Original Research Article

\title{
Students' attitudes on personal drug selection exercise in writing prescription
}

\author{
Manish Kumar*, Lalit Mohan, Hitesh Mishra, Akash Chandra, Harihar Dikshit
}

Department of Pharmacology, Indira Gandhi Institute of Medical Sciences, Patna, Bihar, India

Received: 06 November 2017 Accepted: 27 November 2017

*Correspondence to:

Dr. Manish Kumar, Email: manu072@gmail.com

Copyright: (c) the author(s), publisher and licensee Medip Academy. This is an openaccess article distributed under the terms of the Creative Commons Attribution NonCommercial License, which permits unrestricted noncommercial use, distribution, and reproduction in any medium, provided the original work is properly cited.

\begin{abstract}
Background: This study was developed to know the students' views regarding personal drug (P-drug) concept in rational prescription of drugs and also giving them training of creating and using personal drug concept.

Methods: 40 medical students $\left(5^{\text {th }}\right.$ semester $)$ divided in four groups were involved voluntarily in a three phase, questionnaire based and prospective study. In first and second phase students were taught and asked to derive P-drug using different standard text books and Current Index of Medical Specialties (CIMS) by analyzing efficacy, safety, cost and convenience of drugs used for type II diabetes mellitus. Third phase was designed to know the students' perception regarding the exercise and difficulties faced in the process of P-drug selection. It contained demographic and 12 questions with answer using Likert scale.

Results: Students selected biguanide (metformin) as a P-drug in terms of efficacy, safety, cost and convenience. 95\% (36 out of 40) responded in the questionnaire, out of which $92 \%$ (33 out of 36) had given answer with mean score $\geq 4$. Overall median score was 4 and Interquartile Range was 4-5. 89\% (32) strongly agreed that P-dug selection teaching helped them to understand pharmacology better. Majority (83\% or 30) were in favour of introducing P-drug selection exercises in undergraduate pharmacology curriculum.

Conclusions: P-drug selection exercise helped students to understand the differences among various drugs used for the treatment of type II diabetes mellitus and given them a strong foundation for developing rational use of the medicine in their future career as a doctor.
\end{abstract}

Keywords: P-drug, Pharmacology, Prescription writing, Pharmacology curriculum

\section{INTRODUCTION}

Pharmacology, the study of drugs, is mainly taught to $3^{\text {rd }}$, $4^{\text {th }}$ and $5^{\text {th }}$ semester medical students in India. The traditional pharmacology teaching mainly takes place through didactic lectures, in which passive transfer and memorizing of information of drug class and individual compounds occurs. Whereas practical curriculum mainly includes pharmacy practical, animal experimentations, prescription writing and problem based learning (PBL) which has been criticized. So, there is a need to evaluate practical curriculum. ${ }^{1-3}$
Rational use of drugs (RUD) entails that patients should receive medications appropriate to their specific clinical needs, proper dose and duration, with the lowest cost to them and their community. Safe and effective prescribing is based on a sound knowledge of the disciplines of pharmacology which is a major challenge encountered by students. $^{4}$

The above requirements will be fulfilled by the WHO Guide to Good Prescribing which gives medical students a normative model for therapeutic reasoning and prescribing and provides a six-step guide to the process of rational prescribing. ${ }^{5}$ 
1. Define the patient's problem,

2. Specify the therapeutic objective,

3. Choose a (drug) treatment,

4. Write the prescription and start the treatment,

5. Give patient information and warnings, and

6. Monitor the treatment.

One of the key principles of the WHO approach is the division of step 3 into two steps; namely, Step 3a, consider the suitability of a standard (p-drug) treatment for the disease in general, and Step 3b, verify its suitability for the particular patient and alter the drug if necessary. ${ }^{5}$

The WHO Guide to Good Prescribing emphasizes that future doctors should master both steps of the drugselection process. Selecting a p-drug trains student in pharmacological, clinical and epidemiological principles and in collecting and comparing evidence. By considering various treatment alternatives when developing their personal formulary, students are able to choose an alternative drug treatment more rationally in their professional career more critically. ${ }^{5}$

This study was developed to know the students' views or perceptions regarding personal drug concept in rational prescription of drugs and also giving them training of creating and using personal drug concept. So that the outcomes of this study could be used to modify undergraduate pharmacology teaching pattern.

\section{METHODS}

This study was a questionnaire based, prospective, quasiexperimental study without a comparison group and pretest. study was carried out in the Department of Pharmacology was approved from Institutional Ethics Committee of Indira Gandhi Institute of Medical Sciences, Patna, Bihar, India. Forty (40) $5^{\text {th }}$ semester medical students were randomly selected, divided into four groups and each group containing 10 students. Students were informed that their participation in this study will be voluntary. Consent was taken from students. Study was conducted in three phases for 2 months.

In first phase each group was taught for 2 hours regarding P-drug selection on type II diabetes mellitus as per Joshi and Jayawickramarajah by using power point slides, standard text books and handouts with drug costs. Students were also taught about how to analyze and give score $(\alpha)$ to drugs for four parameters like: ${ }^{6}$

1. Efficacy was derived according to the efficacy profile written in standard text books. Drug with more efficacy were given higher score.

2. Safety of a drug was described according to the side effect profile written in standard text books. Drug with more side effects were given lower score.

3. Cost was compared by taking average of costs of different brands written in Current Index of Medical Specialties (CIMS).
4. Convenience was compared according to the availability of drug, dosage form, dosage schedule, route of administration. ${ }^{7}$

Scores were given to each four parameters from 1 to 10 for each drug. Each parameter had given a fractional numerical rating $(\beta)$ according to the importance i.e. 0.4 for efficacy, 0.3 for safety, 0.2 for cost and 0.1 for convenience. Score $(\alpha)$ was multiplied by fractional numerical rating $(\beta)$ to get total score $(\gamma=\alpha \times \beta)$. Higher total score indicated a better value.

In second phase students were asked to derive or select a P-drug among drugs used for the treatment of type II diabetes mellitus.

In third phase students were asked to complete a questionnaire (Annexure 1). This phase was designed to know the students' view or perception regarding the exercise and difficulties faced in the process of P-drug selection. This phase of study consisted of two parts.

1. The first part had demographic related questions and other relevant information.

2. The second part of the questionnaire consisted of 12 statements to know student's point of view regarding personal drug concept ( $\mathrm{P}$ drug concept). The students were asked to score each individual statement and denote their degree of agreement by using a Likerttype scale with the following key: 1-Strongly disagrees, 2-Disagree, 3-Neutral, 4- Agree and 5Strongly agree with the statement.

Students were instructed to use whole numbers only. The questionnaire was based on previous study. ${ }^{[8,9]}$ The reliability of questionnaire was assessed and discussed with the faculty members of the Department of Pharmacology, IGIMS, Patna.

\section{Statistical analysis}

Responses from participants were tabulated. The quantitative data were expressed as median and interquartile range (IQR). Data was analysed through Graph Pad software.

\section{RESULTS}

Out of 40 students/ participants, $60 \%$ were males and $40 \%$ were females. 100\% were Indian. 87.5\% (35) participants had medium of instruction in school was in English language. Out of $40 \%$ (16) only $62.5 \%$ (10) of participants liked mathematics at school. Out of $100 \%$ (40) of participants $90 \%$ (36) liked chemistry at school.

According to Table 1, students selected biguanide (metformin) as a personal drug in terms of efficacy, safety, cost and convenience for the treatment of Type II Diabetes Mellitus. 
Table 1: Selection of personal formulary (P-drug) from drug groups of Diabetes Mellitus type II $(\gamma=\alpha \times$ x $\beta)$.

\begin{tabular}{|llllll|}
\hline Drugs/ drug group & Efficacy $(\mathbf{0 . 4})^{\beta}$ & Safety $(\mathbf{0 . 3})$ & Cost $(\mathbf{0 . 2})$ & Convenience $(\mathbf{0 . 1})$ & Total $(\gamma)$ \\
\hline Sulfonylureas & $7^{\alpha}(2.8)^{\gamma}$ & $6(1.8)$ & $8(1.6)$ & $6(0.6)$ & 6.8 \\
\hline Meglitinide/ phenyllanine analogues & $7(2.8)$ & $6(1.8)$ & $4(0.8)$ & $3(0.3)$ & 5.7 \\
\hline DPP-4 inhibitors & $6(2.4)$ & $6(1.8)$ & $3(0.6)$ & $7(0.7)$ & 5.5 \\
\hline Biguanide & $8(3.2)$ & $8(2.4)$ & $7(1.4)$ & $6(0.6)$ & 7.6 \\
\hline Thiazolidinediones & $5(2.0)$ & $5(1.5)$ & $6(1.2)$ & $8(0.8)$ & 5.5 \\
\hline $\boldsymbol{\alpha}$-Glucosidase inhibitors & $5(2.0)$ & $6(1.8)$ & $5(1.0)$ & $4(0.4)$ & 5.2 \\
\hline
\end{tabular}

Table 2: Student's view/perception on personal drug selection exercise.

\begin{tabular}{|lll|}
\hline Questions & Answers \\
\cline { 2 - 2 } & Median & Interquartile range (IQR) \\
\hline $\begin{array}{l}\text { P-drug concept gives in depth knowledge about drug, classification to which } \\
\text { drug belongs and other drugs in this group }\end{array}$ & 5 & $4-5$ \\
\hline $\begin{array}{l}\text { While comparing the different drugs in same group the intragroup } \\
\text { variability is highlighted }\end{array}$ & $3-4$ \\
\hline $\begin{array}{l}\text { As compared to prescription writing, P-drug concept helps in selecting a } \\
\text { drug for a disease rather than for a patient }\end{array}$ & 4 & $4-5$ \\
\hline $\begin{array}{l}\text { P-drug concept will have a long-term impact on your mind as compared to } \\
\text { prescription writing }\end{array}$ & 4 & $3-4$ \\
\hline P-drug gives a better idea of cost of different drugs in same group & 3 & $2-4$ \\
\hline $\begin{array}{l}\text { Teaching of P-drug concept is a two-way learning process where students } \\
\text { get equal chance to present their view }\end{array}$ & 3 & $3-4$ \\
\hline $\begin{array}{l}\text { Some difficult topics which are not properly covered in theory are better } \\
\text { understood. }\end{array}$ & 4 & $4-5$ \\
\hline Selection of P-drug is a time-consuming process & 5 & $5-5$ \\
\hline P-drug can be differing from drug of choice & 4 & $3-4$ \\
\hline $\begin{array}{l}\text { Comparing efficacy is more difficult than comparing safety while selecting } \\
\text { a P-drug }\end{array}$ & 4 & $4-4$ \\
\hline $\begin{array}{l}\text { Using CIMS, textbook and reference books is helpful in selection of a P- } \\
\text { drug }\end{array}$ & 5 & $4-5$ \\
\hline $\begin{array}{l}\text { P-drug selection exercises can be included in undergraduate pharmacology } \\
\text { practical curriculum }\end{array}$ & 5 & $4-5$ \\
\hline
\end{tabular}

95\% (36 out of 40) participants responded in the questionnaire, out of which $92 \%$ (33 out of 36) had given answer with mean score $\geq 4$. Overall median score was 4 and Interquartile Range was 4-5 (IQR 4-5). 89\% (32) students strongly agreed that P-dug selection teaching helped them to understand pharmacology better. Majority (83\% or 30 ) of students were in favour of introducing the process of selection of P-drug in undergraduate pharmacology curriculum.

\section{DISCUSSION}

Irrational prescribing is a common problem which can be prevented..$^{10}$ Training of students in pharmacology should be in such a way that they are able to prescribe rationally for common diseases, calculate dosages depending upon age and sexes and prevailing health status of individuals, administer the drug through appropriate route and also identify adverse drug reactions and interactions. ${ }^{11}$ There is a need of developing patient oriented problem-solving system of teaching in pharmacology in which students are oriented to learn rational use of drug by proper training. ${ }^{12}$ A survey in medical school in United Kingdom has revealed that medical student feels the need for more teaching of therapeutics in undergraduate medical curriculum. ${ }^{13}$

Eventually, what really matters is the quality of therapeutic reasoning and prescribing skills. Currently, these skills are inadequate. The prescription writing exercises are no more than a recall test and they hardly ensure the art of scientific prescribing. Although the clinical pharmacology exercises sensitize the students for a rational use of drugs, it is not known to what extent they improve the prescribing skill. Unfortunately, these skills are not reinforced during clinical postings and so their practical application remains incomplete. $^{1}$

In 1994, a manual on the principles of rational prescribing called 'Guide to Good Prescribing' was developed by the 
World Health Organization (WHO) Action Program on Essential Drugs. ${ }^{14}$ In 2001, 'Teachers' Guide to Good Prescribing' was developed as a companion volume to help medical teachers better use the 'Guide to Good Prescribing' to teach undergraduate medical students. Students are taught to develop a standard treatment for common disorders and a set of first-choice drugs called Personal or P-drugs. Students should develop their set of P-drugs using National and International treatment guidelines, formularies, textbooks and other sources of drug information. ${ }^{15} \mathrm{~A}$ six-step problem solving approach is used to apply this set of P-drugs to specific patient problems.

Rai has recommended inclusion of P-drug concept in the undergraduate pharmacology practical curriculum. ${ }^{16}$ Evaluation of teaching by the use of questionnaire to students is now common. ${ }^{17}$

In first phase of this study, students were taught how to define their own P-drugs for common disorders by using National and International treatment guidelines, formularies, textbooks and other sources of drug information and were encouraged to develop a personal formulary, so that they can be good future prescribers. These were according to the previous study and WHO's principles of rational prescribing called 'Guide to Good Prescribing'. $9,14,18$

In second phase of this study, students had given high score to Biguanide (Metformin) in terms of efficacy and safety. In terms of cost and convenience, DPP-4 inhibitors were given high scores compared to other drugs by students. But the cumulative score of Biguanide (Metformin) was higher than other drugs. So, after discussion Biguanide (Metformin) with highest score was selected as P-drug for type II diabetes mellitus (Table 1).

In this study, questionnaire (Annexure 1) had been distributed to know students' opinion on the usefulness of $\mathrm{P}$ drug concept approach, relevance of in understanding a particular topic and prescribing drug. We followed the method described by Joshi and Jayawickramarajah in which four criteria - efficacy, safety, cost and convenience were considered while selecting a P-drug. ${ }^{6}$

Students agreed that P-drug concept had imparted a clear concept while choosing drug for prescription writing and given different reasons that the process of $\mathrm{P}$-drug selection is interactive and interesting way of learning (Table 2, question no 3). This concept had enabled them to discriminate between different drugs while prescribing (Table 2, question no 1 and 2).

P-drug concept helps in selecting a drug for a disease rather than for a patient because P-drugs are our priority choice for the given indication. ${ }^{19}$ But during this study students thought that P-drugs are drugs personal to the patients, rather than personal to the doctor (Table 2, question no 4).
Drug selection process had given idea to students about cost of the drugs which was not taught in conventional prescription writing exercises (Table 2, question no 5). Teaching of $\mathrm{P}$-drug concept is a two-way learning process i.e. it is an interactive session in which students discuss with their group members as well as with teachers in comparison to didactic lectures. So, in such methods students get equal chance to present their view (Table 2, question no 6).

Some difficult topics which are not covered in theory or for those topics where time allotment is less are better understood most of the students were neutral for this statement (Table 2, question no 7). Students agreed that selection of P-drug was a time-consuming process (Table 2 , question no 8).

P-drug is different from drug of choice- students' opinion varied from neutral to agree. P-drug can be different from drug of choice. Drug of choice is mainly selected by evidence based medicine and the experience of physicians, whereas P-drug is selected on different criteria i.e. efficacy, safety, suitability and cost (Table 2, question no 9).

Students' opinion varied from neutral to agree in interquartile range for question (Table 2, question no 10) stating that the comparing efficacy is more difficult than comparing safety while P-drug selection. This is due to the fact that most of the textbooks highlight side effects more than the efficacy. Also, students should not confuse the term efficacy with potency of a drug. Students were agreed that using CIMS, textbook and reference books was helpful in selection of a P-drug (Table 2, question no 11). Students agreed P-drug selection exercises should be included in undergraduate Pharmacology practical curriculum (Table 2, question no 12).

The basic motive of teaching P-drug concept is that instead of memorizing one can develop personal formulary after proper discussion and know how to prescribe rather than what to prescribe. In a recent study on P-drug, students were of the opinion that process of P-drug selection helped them to understand pharmacology better. ${ }^{20}$

\section{CONCLUSION}

P-drug selection exercise helped students to understand the differences between various drugs used for the treatment of type II diabetes mellitus. Teaching P-drug selection had given them a strong foundation for developing rational use of the medicine in their future career as a doctor. Also, it will be helpful in modifying undergraduate pharmacology teaching pattern in future.

\author{
Funding: No funding sources \\ Conflict of interest: None declared \\ Ethical approval: The study was approved by the \\ Institutional Ethics Committee
}




\section{REFERENCES}

1. Desai M. Changing face of pharmacology practicals for medical undergraduates. Indian $\mathrm{J}$ Pharmacol. 2009;41:151-2.

2. Gitanjali B. New wine in new bottles. Indian J Pharmacol. 2004;36:63-4.

3. Hatiharan TS. Need For changes in the practical pharmacology curriculum of medical undergraduates. Indian J Pharmacol. 2004; 6:181.

4. Maxwell SRJ, Daniel S, McQueen, Ellaway R. e Drug: a dynamic interactive electronic drug formulary for medical students. $\mathrm{Br} \mathrm{J}$ Clin Pharmacol. 2006;62(6):673-81.

5. De Vries TPGM, Daniels JA, Mulder CW, Groot OA, Wewerinke L, Barnes KI, et al. Should medical students learn to develop a personal formulary? An international, multicentre, randomised controlled study. Eur J Clin Pharmacol. 2008;64:641-6.

6. Joshi MP, Jayawickramarajah PT. A problemorientated pharmacotherapy package for undergraduate medical students. Med Teach. 1996;18:75-6.

7. Singh NR. P-drug concept and the undergraduate teaching. Indian J Pharmacol. 2008;40:285.

8. Shankar PR, Palaian S, Gyawali S, Mishra P, Mohan L. Personal drug selection: Problem-based learning in pharmacology: Experience from a medical school in Nepal. PLoS One. 2007;6:e524.

9. Mohan L, Chogtu B, Adiga S, Shenoy S, Bairy KL. Kishore A. Undergraduate medical students' perception regarding personal drug selection exercise. IJPCS. 2012; 1(2):61-7.

10. Walley $\mathrm{T}$, Bligh $\mathrm{B}$. The educational challenge of improving prescribing. Postgraduate Education for General Practice. 1993;4:50-4.

11. Ruckmani A. The role of pharmacologist; present and future, Indian J Pharmacol. 2006;138(2):145-6.
12. Bapna JS. Experiences in Teaching Rational Drug Use. Indian J Pharmacol. 1993;25:2-4.

13. Ward F, Miolszweski K. Evaluation of the impact of pharmacist-led therapeutic tutorials on third-year medical students' knowledge and understanding of drugs used in clinical practice. Med Teach. 2002;24:628-33.

14. De Vries TPGM, Henning RH, Hogerzeil HV, Fresle DF Guide to good prescribing. Geneva: World Health Organization. 1994:14.

15. Hogerzeil HV, Barnes KI, Henning RH, Kocabasoglu YE, Moller H, Smith AJ, et al. Teachers' guide to good prescribing. Geneva: World Health Organization. 2001:98.

16. Rai J. Recommendations for Undergraduate Pharmacology Practical Curriculum (For attention of MCI). JK Practitioner. 2006;13:175-6.

17. Metcalfe DH, Mathrau M. Students perception of good and bad teaching: a report of a critical incident study, Med Edu. 1995;29:193-7.

18. Mohan L, Manish, Bairy KL, Babu M, Amberkar V, Narayanareddy $M$, et al. Personal formulary for anxiety disorder developed by post graduates of pharmacology. Int $\mathbf{J}$ of Phar Sciences Review and Research. 2011;7(1):019.

19. De Vries TPGM, Henning RH, Hogerzeil HV, Fresle DF. Guide to good prescribing. Geneva: World Health Organization; 1994:134.

20. Devi V. Teaching P-drug selection; experiences from medical school in India. Int J Pharmacol and Clin Sci. 2012;1:9-14.

Cite this article as: Kumar M, Mohan L, Mishra H, Chandra A, Dikshit H. Students' attitudes on personal drug selection exercise in writing prescription. Int J Basic Clin Pharmacol 2018;7:14752. 


\section{Annexure 1: Questionnaire to know the student's view on personal drug selection in writing prescription.}

Participation depends on your willingness. No personal information should be written on the paper (name, registration number). Please answer legibly and write the appropriate no. in boxes wherever required.

\section{Part 1- Demography}

Sex: M/ F

Nationality:

Medium of instruction at school: English/ Regional

Was Mathematics one of your subjects at school $(10+2$ level $)$ ? Yes/ No

Your attitude towards Mathematics at school: Liked it/Neutral/Hated it

Your attitude towards Chemistry at school: Liked it/Neutral/Hated it

Government selected/ self-financing

Part 2- For the following statements score using the following key: 1- strongly disagree, 2- disagree, 3neutral, 4-agree, 5-strongly agree

1. P-drug concept gives in depth knowledge about drug, classification to which drug belongs and other drugs in this group

2. While comparing the different drugs in same group the intragroup variability is highlighted

3. As compared to prescription writing, P-drug concept helps in selecting a drug for a disease rather than for a patient

Give reasons

4. P-drug concept will have a long-term impact on your mind as compared to prescription writing Give reasons.

5. P-drug gives a better idea of cost of different drugs in same group

6. Teaching of P-drug concept is a two-way learning process where students get equal chance to present their view

7. Some difficult topics which are not properly covered in theory are better understood.

8. Selection of P-drug is a time-consuming process

9. P-drug can be differing from drug of choice Give reasons with example

10. Comparing efficacy is more difficult than comparing safety while selecting a P-drug

11. Using CIMS, textbook and reference books is helpful in selection of a P-drug

12. P-drug selection exercises can be included in undergraduate pharmacology practical curriculum 